\title{
Meta
}

Journal des traducteurs

Translators' Journal

\section{CARDINAL, Linda et LAROCQUE, François, dir. (2017) : La Constitution bilingue du Canada : un projet inachevé. Prisme. Québec : Presses de l’Université Laval, 319 p.}

\section{Catherine Zekri}

Volume 64, numéro 1, avril 2019

URI : https://id.erudit.org/iderudit/1065341ar

DOI : https://doi.org/10.7202/1065341ar

Aller au sommaire du numéro

Éditeur(s)

Les Presses de l’Université de Montréal

ISSN

0026-0452 (imprimé)

1492-1421 (numérique)

Découvrir la revue

Citer ce compte rendu

Zekri, C. (2019). Compte rendu de [CARDINAL, Linda et LAROCQUE, François, dir. (2017) : La Constitution bilingue du Canada : un projet inachevé. Prisme.

Québec : Presses de l’Université Laval, 319 p.] Meta, 64(1), 293-296.

https://doi.org/10.7202/1065341ar d'utilisation que vous pouvez consulter en ligne.

https://apropos.erudit.org/fr/usagers/politique-dutilisation/ 
translation "where the target audience [...] may not possess the required, source culture and language related background knowledge to be able to understand the text" (p. 170). She therefore suggests that "changes in the topical structure of this component may [...] make processing more difficult and endanger the correct/adequate interpretation of the translation." I would have thought that the opposite would be true: changes are particularly frequent in the background section because it is here that the differences between the source and target readers' knowledge would be the greatest and these changes in fact augment the reader's comprehension of the text.

The final data chapter examines rhetorical structure through an analysis of the frequency, types and position of relational propositions. Once again, the quantitative results show no statistically significant difference between the source and target texts (p. 193). Qualitative analyses, on the other hand, do reveal some minor differences in both the type and position of relational propositions and it is interesting that "only eight of the 20 texts have completely identical rhetorical structures" (p. 196). Károly finds that shifts occur most frequently in the commentary section of the news stories and this, she argues, means that "consciously or unconsciously [...] translators modify the relational propositional content of this component and thus, possibly, very subtly alter the intended message" (p. 200, author's italics).

The eighth and final chapter brings the results of each of the data analysis chapters together. It reiterates the main finding across all of the chapters, namely that "[i]t is [...] not so much the frequency, but rather the quality (the type, the location, the optional/automatic nature) of the shifts that produce changes at the various levels of discourse structure" (p. 213). As regards translation universals, there was no statistically significant evidence in support of either of the two main universals under consideration here (the explicitation hypothesis and the repetition avoidance hypothesis). However, Károly does rather go out on a limb, despite all the hedging, when she concludes, based on her study, that "shift of coherence as a phenomenon is special to the process of translation and may thus (possibly) be regarded as a translation universal" (p. 212, author's italics).

Aspects of Cohesion and Coherence in Translation: The case of Hungarian-English news translation is certainly a useful contribution to the field of translation studies. Károly's main contribution is methodological: she shows that it is possible to test empirically what happens to cohesion and coherence in translation. She has devised a model that other scholars can now use to explore the same phenomena in different corpora of translated texts.
Her quantitative work is a particular strength: she is meticulous about reporting the results of statistical significance tests, and it is particularly laudable that she can quantify the validity of the manual coding by having each analysis performed by two different researchers. The main drawback of the study is without doubt the small size of the research corpus which unfortunately makes it difficult to know whether Károly's conclusions really are a reflection of the wider reality. Readers will no doubt be convinced that there are qualitative shifts affecting cohesion and coherence in translation. However, the very small number of texts in the corpus means that it is impossible to know whether the lack of statistically significant differences in the frequency of the shifts is a true reflection of reality.

Mairi Mclaughlin University of California, Berkeley, United States of America

CARdinal, Linda et LARocque, François, dir. (2017): La Constitution bilingue du Canada: un projet inachevé. Prisme. Québec: Presses de l’Université Laval, 319 p.

Il est inédit que la traduction se trouve au cœur d'une impasse constitutionnelle. L'ouvrage collectif La Constitution bilingue du Canada: un projet inachevé, d'un intérêt remarquable pour plusieurs disciplines: traduction, sociolinguistique, science politique, droit constitutionnel, entre autres, éclaire sous plusieurs angles le très important (et épineux) dossier de la traduction de la Constitution canadienne et secoue la poussière qui s'y est déposée par couches successives.

Comment un pays démocratique officiellement bilingue et doté d'une vigoureuse industrie de la traduction, stimulée en partie par le bilinguisme institutionnel de sa fonction publique fédérale, peut-il ne pas disposer d'une version française officielle de ses textes constitutionnels? C'est pourtant la situation invraisemblable dans laquelle se trouve toujours empêtré le Canada en 2019. Quels en sont les enjeux juridiques, politiques, identitaires? Pourquoi, comment les textes constitutionnels du Canada ont-ils pu échapper à la traduction? En quoi consistent ces textes fondateurs? Quelle en est l'importance? Et surtout, comment remédier à cette défaillance lourde de conséquences alors que les provinces se sentent peu concernées? Sans être exhaustif, cet ouvrage éclaire ces questions sous plusieurs angles.

Sous la direction de Linda Cardinal et de François Larocque, désireux d'éperonner le débat, douze «témoins privilégiés des négociations constitutionnelles des années $1980 »$ (p. 2) 
appréhendent l'unilinguisme paradoxal de la loi suprême du Canada. D’horizons variés, ces auteurs offrent tantôt un éclairage historique ou politique, tantôt un récit critique des efforts déployés ou des défaillances politiques qui ont paralysé ce projet. D'autres auteurs encore font état des tenants et aboutissants législatifs et des obstacles récurrents, qu'il faudra un jour surmonter s'il est souhaité d'honorer la Constitution canadienne.

Cet ouvrage opère ainsi un retour sur les défis théoriques, politiques, législatifs et identitaires, mais aussi traductionnels que pose la bilinguisation des textes constitutionnels afin d'achever le rapatriement constitutionnel du Canada, mais aussi sur des pistes de solution (pour la plupart déjà empruntées en vain). Encore faut-il s'entendre sur ce qu'on veut dire par traduction; d'éventuels glissements sémantiques préoccupent au plus haut point les parties prenantes et la Constitution repose sur un siècle et demi de jurisprudence de tradition britannique.

L'ouvrage, publié opportunément l'année des 150 ans de la Loi constitutionnelle de 1867, se déroule en quatre temps: une introduction donnant le ton aux trois parties qu'elle annonce. Dans le premier temps, L. Cardinal et F. Larocque exposent la situation, son contexte et ses enjeux, en soulignant l'embarras dans lequel se trouve figée la Constitution bilingue inachevée. Ils rappellent que, malgré l'enchâssement du caractère officiel du français et de l'anglais dans la Loi constitutionnelle de 1982 , qui, en vertu de l'article 55, exige que soit élaborée et adoptée "dans les meilleurs délais» (p. 1) la version française des textes constitutionnels, le gouvernement canadien n'avait pas encore procédé 35 ans plus tard. Ils soulignent l'absence de volonté politique et l'incohérence constitutionnelle à laquelle donne lieu l'unilinguisme de la plupart des textes constitutionnels, y compris la fondatrice Loi constitutionnelle de 1867.

Dans un second temps, la deuxième partie réunit trois chapitres qui procurent une mise en contexte étayée. Le premier chapitre, par Sébastien Grammond, retrace les origines historiques du bilinguisme législatif au Canada. L'auteur en tire le constat que la traduction française de la loi est très tôt devenue un acquis et que, au-delà de l'égalité de statut des langues revendiqué par l'élite politique francophone dans les années 1840-1850, c'est l'égalité des peuples qui était recherchée. Dès l'adoption de la Loi constitutionnelle de 1867 à Londres, pièce maîtresse de la Constitution canadienne antérieure à 1982, adoptée en anglais seulement, des traductions officieuses voient le jour, peu discordantes mais néanmoins idiosyncrasiques, si bien qu'il existe plusieurs versions françaises, dont la «version Dorion». Outre les lois constitutionnelles de 1867 et de 1982, une trentaine de textes ont été adoptés au fil des décennies, dont certains, adoptés par le Parlement canadien, sont officiellement bilingues en vertu de l'article 133 de la Loi constitutionnelle de 1867.

Grammond nous renseigne sur les travaux du Comité de rédaction constitutionnelle française, chargé de la traduction et du dépôt pour adoption. La position ontologique du comité, souscrivant aux nouveaux principes fédéraux de rédaction française des lois, l'amena à retraduire les textes de manière à préserver la cohérence conceptuelle et interne des textes, s'éloignant nettement des versions officieuses, employées couramment. Ainsi, par souci d'exactitude traductionnelle, le comité révisa et modernisa les textes constitutionnels, soulevant de toutes nouvelles questions d'ordre théorique: comment surmonter l'anachronisme stylistique, lexical et légal? La dissonance étant inéluctable, laquelle choisir: entre le texte français et son contexte historique, entre le texte constitutionnel et la législation contemporaine ou entre les textes anglais ou français? L'usage historique de la terminologie confère à celle-ci des acceptions légitimes qu'aucune version contemporaine ne saurait rendre. Qu'en serait-il de l'égalité de statut des deux versions alors que l'interprétation juridique repose moins sur le texte que sur la jurisprudence, unilingue anglaise par tradition?

Le deuxième chapitre, par Mary Dawson, ancienne sous-ministre déléguée, Droit public, du ministère de la Justice, relate qu'il revenait au Bureau de la traduction de préparer les versions françaises jusqu'aux années 1970, sans l'intervention d'avocats francophones. Pour pallier cette situation (on devine qu'il s'agit de lacunes en connaissances juridiques) et l'absence de rédacteur francophone à la Section de la législation, un premier avocat francophone fut embauché pour valider la qualité linguistique et la fidélité des traductions. En 1977, le commissaire aux langues officielles Keith Spiker, préoccupé par le traitement des versions françaises, demanda qu'on traitât les deux versions de manière uniforme. "Dès 1980, les traducteurs ne faisaient plus partie du processus» (p. 51), un système à deux corédacteurs fut privilégié.

Dawson retrace les différents stades et rapports du Comité de rédaction constitutionnelle française, ainsi que ses multiples efforts infructueux pour qu'aboutisse l'adoption de la version française. Elle s'interroge sur la possibilité d'un jour respecter les dispositions de l'article 55. Hugo Choquette, au troisième chapitre, défend quant à lui la version Dorion, traduction originale réalisée en 1867 par le chef du Bureau de la traduction et avocat de formation, rédacteur habile et érudit, issu d'une famille incarnant la dualité linguistique du Canada. Très critique du projet de traduction 
de la Constitution et du Comité, il soutient que c'est la fidélité à l'original de la traduction de Dorion qui gêne, par son respect minutieux du style ampoulé et redondant propre à la tradition législative anglaise de la common law de l'époque, à laquelle appartient le British North America Act :

Dorion, juriste francophone et civiliste, avait bien conscience que le document qu'il traduisait n'était pas un texte législatif du droit civil; qu'il provenait d'une tout autre tradition. C'est par respect pour les normes de cette tradition qu'il a voulu être si fidèle au style de l'original. (p. 67)

Cette «phraséologie pompeuse» (p.X), ce «verbiage» (p.X) reflètent, selon Choquette, la tradition du droit public anglais, que partagent les francophones canadiens quoi qu'on en pense.

Sous le thème plus technique de la voie juridique et en mobilisant certains extraits, la deuxième partie rassemble trois chapitres autour du devoir, de l'obligation et du pouvoir de légiférer. Des multiples considérations exposées par les auteurs de ces sections, nous en retenons quatre. Premièrement, l'obligation de faire de la loi suprême du Canada un texte intégralement bilingue, prévue à l'article 55 , n’a jamais été remplie; il est urgent qu'elle le soit à la lumière des dispositions de la Charte canadienne des droits et libertés enchâssée à même la Loi constitutionnelle de 1982.

Deuxièmement, l'unanimité des provinces est inévitablement requise à l'adoption de l'intégralité de la Constitution en français, éventualité peu probable et contraire au caractère obligatoire de l'article 55. Troisièmement, traduire par morceaux plusieurs dispositions constitutionnelles n'ayant pas encore de version francisée officielle permettrait de contourner partiellement l'unanimité des provinces. En outre, compte tenu de l'obligation de rédiger les lois dans les deux langues, toute «modification constitutionnelle n'ayant pas pour objectif l'adoption de la version française de la Constitution contribue néanmoins à la réalisation de cet objectif» (p. 169). Enfin, cette deuxième partie se penche ici et là sur le positionnement du Québec. Elle met aussi en lumière l'inaction des ministres de la Justice fédéraux qui se sont succédé et la divergence d'intérêt politique entre les provinces sur la question fondamentale du bilinguisme canadien.

La partie finale, dans le quatrième temps, s'étend sur deux chapitres brefs mais percutants qui explorent le passage obligé de la voie politique. Au chapitre sept, W. Newman fait remarquer qu'en misant et sur la collaboration fédérale-provinciale et sur les exigences juridiques, l'article 55 appelle des mécanismes politiques plutôt que juridiques. Il dresse par ailleurs la liste des dix-sept textes constitutionnels pour lesquels il n'existe pas encore de version française ayant force de loi, et des sept pour lesquels il en existe une.

Pour Serge Joyal, dont le chapitre couronne ce collectif, si l'article 55 est un objectif incontournable, il n'en demeure pas moins que l'avenue à suivre pour y parvenir est semée d'embûches et sans issue. La Loi constitutionnelle de 1982 visait explicitement à résoudre l'incongruité de l'unilinguisme officiel dans un pays officiellement bilingue, léguée par l'ancien British North America Act en 1867, en donnant une portée juridique identique aux versions française et anglaise. Mais la passivité historique du ministère de la Justice remontant au-delà de la Confédération de 1867 et «aussi loin qu'à la première Chambre d'assemblée formée à Québec en 1791 »(p. 200) est flagrante, notamment sous le gouvernement Trudeau de 1971. Le projet de modification constitutionnelle, à l'occasion du rapatriement de la Constitution canadienne, omettait alors d'accorder la priorité à la dotation d'une constitution bilingue, malgré l'urgence politique que revêtait à cette époque la reconnaissance des francophones du Canada. La collaboration politique qu'engage l'article 55 semble relever de l'utopie:

Sans leadership politique solide comme le roc, il n'est pas réaliste de croire qu'on réunira les 11 gouvernements du pays pour faire du français la langue de la Constitution. On touche là à ce qu'il y a de fondamentalement irréaliste dans le libellé de l'article 55: d'abord soumettre à la règle de l'unanimité du fédéral et des provinces l'adoption de la version française de toutes les dispositions de la Loi constitutionnelle de 1867. La barre à atteindre est en fait inaccessible et il ne faut jamais minimiser l'intérêt divergent des provinces, non plus que le programme politique concurrentiel qui peut exister entre les chefs politiques du fédéral et de l'une ou l'autre province lorsque les allégeances politiques et les intérêts partisans diffèrent. (p. 206)

Soulignons que sont annexées les versions «Dorion» et du Comité de rédaction constitutionnelle française, ainsi que la clairvoyante allocution de clôture prononcée par l'ancien commissaire aux langues officielles Graham Fraser en 2015 à Ottawa à l'occasion du colloque Une constitution officielle bilingue au Canada en 2017?

Outre quelques opinions discordantes entre les auteurs, le doute soulevé en introduction par Cardinal et Larocque semble justifiable:

[...] les membres de la classe juridique se rendraient-ils complices des acteurs politiques frileux lorsqu'ils ont recours aux diverses 
versions françaises [non officielles] des textes constitutionnels en reconnaissant du même souffle que celles-ci n'ont aucune force sur le plan juridique? (p. 5)

Selon ces derniers, l'officialisation de la langue française aux côtés de la langue anglaise, banalisée par les élus bien qu'exigée par la Constitution, est requise afin d'accorder au peuple francophone la reconnaissance de sa légitimité politique, laquelle repose sur le principe fondateur de la dualité linguistique. Lui dénier ce droit prolonge et accentue l'injustice. Pendant que le Canada semble attendre qu'une menace pèse sur «l'intégrité du pays [...] qui inciterait la classe politique à officialiser la version française de la Constitution canadienne» (p. 5), suggère l'ouvrage, la «grave erreur» (p. 208) de l'article 55 continue d'alimenter le clivage linguistique. Mentionnons pour finir que l'ouvrage laisse entrevoir de multiples pistes de recherche dont certaines concernent la discipline de la traduction.

Catherine ZeKri École nationale d'administration publique, Montréal, Canada

Cordingley, Anthony and Frigau Manning, Céline, eds. (2017): Collaborative Translation: From the Renaissance to the Digital Age. London/ New York: Bloomsbury Academic, 272 p.

The Bloomsbury Advances in Translation series is edited by Jeremy Munday, director of the Centre for Translation Studies at the University of Leeds. The first volume was published in 2011, and this book represents its twelfth publication. With a focus on translation practices, processes and theory, the series has increased its pace to match the progress in Translation Studies research, with three more books having followed this one in 2017 alone. Other translation topics covered in the series include training, music and poetry, retranslation and adaptation, as well as cultural and institutional translation.

The editors of Collaborative Translation: From the Renaissance to the Digital Age, both Associate Professors at the Université Paris 8, bring unique backgrounds to the project. Cordingley publishes in the area of self-translation, having served as editor of a text (Cordingley 2013) in a related series called Bloomsbury Studies in Translation. Manning focuses on translation of the arts, namely theatre and opera, writing on this subject for various journals including Opera Quarterly and Nineteenth-Century Music. It is therefore not surprising that one third of the book is devoted to author-translator collaboration, and several chap- ters deal with translating comedies and poetry. Unfortunately, this is done at the expense of other topics that one might expect in a book about collaborative translation, such as the efficacy of various collaborative techniques, or the various roles present within a translation team. Nevertheless, valuable material is presented in relation to several aspects of collaboration, with each chapter being separately authored.

The introduction establishes a clear definition of collaborative translation. It is validated as being a legitimate and positive activity, the wartime perceptions of "collaboration with the enemy" being long outdated. Not only has it existed for many centuries, but collaboration was actually the norm for both translation and writing until the Renaissance. At that time, it became a hidden activity as a result of the prevailing "myths of singularity" (p. 4) which attributed genius to a single author. The advent of copyrights only reinforced the practice of labelling a text with a single name rather than recognizing the teamwork involved in most creative endeavours. The recent interest in collaborative translation signals a return to a historic activity and an acknowledgement that it has always existed. The editors conclude that a translation can be called collaborative if there is consistent interaction throughout the process, whether simultaneous or successive. This can include the input of authors, advisors, editors, and consultants.

Section 1 expounds on the visibility of collaborative translation throughout history. Belén Bistué posits that in the $15^{\text {th }}$ century, Leonardo Bruni was instrumental in suppressing its value, by the simple fact that he excluded it from his treatise ${ }^{1}$ about correct translation (Viti 2004). His reflection on translation is considered by some to be hypocritical since he emphasized excellence in both source and target languages, yet used Latin words to refer to Greek writings, unlike other authors of his time. Centuries later, collaborative translation made a comeback in an area to which it is well suited, perhaps even necessary: theatrical translation. Françoise Decroisette recounts how in 1993, the European Goldoni Association gathered together twenty translators to produce a shared translation of forty untranslated comedies written by Carlo Goldoni. The collaboration involved not only these translators, but also actors, directors and spectators who gave feedback to help form the finished product.

During this time, a longer-running project was in progress from 1987 to 2000, involving collaborative translation of texts by Guicciardini, Savonarola and Machiavelli. Chapter authors JeanLouis Fournel and Jean-Claude Zancarini discuss how the guiding rule was that "the translated 